\title{
Tapa, étoffes cosmiques de l'Océanie dirigé par Laurent Guillaut et al.
}

\section{Raymond MAYER}

\section{(2) OpenEdition}

\section{Journals}

\section{Édition électronique}

URL : http://journals.openedition.org/jso/6165

DOI : $10.4000 /$ jso.6165

ISSN : $1760-7256$

\section{Éditeur}

Société des océanistes

\section{Édition imprimée}

Date de publication : 15 décembre 2010

Pagination : 267-269

ISBN : 978-2-85430-027-7

ISSN : 0300-953x

\section{Référence électronique}

Raymond MAYER, «Tapa, étoffes cosmiques de l'Océanie dirigé par Laurent Guillaut et al. », Journal de la Société des Océanistes [En ligne], 130-131 | 2010, mis en ligne le 15 décembre 2010, consulté le 21 septembre 2020. URL : http://journals.openedition.org/jso/6165 ; DOI : https://doi.org/10.4000/jso 6165 
générale des musées australiens et le cadre social et politique de leur développement.

Ces réserves ne sauraient atteindre l'excellente étude de B. Craig (« Edgar Waite's north-west Pacific expedition - the hidden collections », pp. 173-195) sur la campagne menée par le directeur du South Australian Museum d'Adelaide entre mai et septembre 1918 dans les anciennes possessions allemandes (NouvelleGuinée et archipel Bismarck) restées sous administration militaire australienne jusqu'à la fin de la Première Guerre mondiale et les traités qui s'ensuivirent. Les objets que Waite acquit auprès de Blancs vivant dans leurs plantations (comme celles de Pettersson à Tabar) ou installés dans les villes (tel le commerçant Whiteman de Rabaul) relèvent évidemment de la «collecte coloniale » au sens de Chr. Gosden et Ch. Knowles, mais leur ancienneté et leur facture, autant que la laissent apercevoir les reproductions, semblent justifier qu'ils soient mieux étudiés et mis en valeur. Il faut sans doute en dire autant des objets collectés par Williams, qu'évoque moins en détail S. Schaffarczyk (" $A$ Rara Avis : FE Williams, the Government Anthropologist of Papua, and the Official Papuan Collection ", pp. 198-220), et plus récemment par Moriarty (N. Wilson, " (Works) of paradise ant yet : Stanley Gordon Moriarty, Tony Tuckson and the collection of Oceanic Art at the Art Gallery of New South Wales ", pp. 222241), ou par les collecteurs travaillant pour les autorités culturelles australiennes ( $\mathrm{S}$. Cochrane, « $\mathrm{Mr}$ Pretty'Predicament : Ethnic Art Field Collectors in Melanesia for the Commonwealth Arts Advisory Board, 1968-1973 », pp. 244-274).

On mentionnera encore, à propos des collecteurs et collectionneurs, les souvenirs d'H. Beran (« Recollections of a Massim Art Collector », pp. 290-304), et la description sommaire que fait Chr. Dixon d'objets océaniens ayant appartenu à Max Ernst avant d'être achetés en 1985 par la National Gallery of Australia («Max Ernst, artist and collector», pp. 276-288). Mais à côté de ces contributions qui feront consulter cet ouvrage pour son intérêt historique (avec un index utile, présentant néanmoins un décalage d'une à dix pages par rapport au texte à partir de la p. 222, et non constamment « deux » comme assure la liste d'errata), il faut signaler l'importance spéciale que peuvent avoir non seulement pour les Océanistes, mais pour les habitants du Pacifique, les cinq articles de la dernière section, « The Collections » : «Who is collecting Pacific Island Archives in Australia now ? », d'E. Maidment (pp. 308-325), «The Pacific collections of the National Library of Australia ; a reflection of national awareness and perception of the Pacific region" de $\mathrm{S}$. Woodburn (pp. 328-344), « The Banaba-Ocean Island Chronicles: Private collections, indigenous record keeping, fact and fiction » de K. Raobeia-Sigrah et St. King (pp. 346-363), « Pacific collections in the National Museum of Australia » de D. Kaus (pp. 366-383) et « Pacific focus ; bringing knoledge about photographic collections in Australia to Pacific communities » de T. Antsoupova et E. Maidment (pp. 386-401). S. Woodburn montre en particulier que si l'Australie a pu connaître des périodes de repli et négliger parfois ses collections de documents et d'objets du Pacifique, se développait dans le même temps sa « conscience nationale » de grande puissance régionale, menant ultérieurement à l'étude à nouveaux frais et à la mise en valeur de ce patrimoine considérable, la plaçant aujourd'hui au rang des « grandes puissances » sur le plan culturel, du moins en Océanie.

\section{RÉFÉRENCES CITÉES}

BiskuP Peter (ed.), 1974. The New Guinea Memoirs of Jean Baptiste Octave Mouton, Honolulu, The University Press of Hawaii, Pacific History Series 7.

Finsch Otto, 1888. SamoaFahrten, Reisen in Kaiser Wilhelms-Land und Englisch-Neu-Guinea... an Bord des deutschen Dampfers "Samoa », Leipzig, Hirt \& Sohn.

Gosden Chris and Chantal Kwnoles, 2001. Collecting Colonialism. Material Culture and Colonial Change, Oxford-New York, Berg.

O'Hanlon Michael and Robert L. Welsch (eds), 2000. Hunting the Gatherers. Ethnographic Collectors, Agents and Agency in Melanesia, 1870s1930s, New York-Oxford, Berghahn Books.

Gilles Bounoure, Paris

Guillaut Laurent, Fanny Wonu Veys, Hélène Guiot et al. 2009. Tapa, étoffes cosmiques de l'Océanie, Cahors, Musée de Cahors Henri-Martin, 128 p., préface, articles et catalogue d'exposition, bibliogr., ill. couleur.

Ce catalogue d'exposition réalisé à Cahors, comme ceux de Chartres en 2000 et 2004, est un exemple parfait d'édition d'envergure mondiale réalisée à partir d'une initiative locale. Foin des hégémonies des rédactions scientifiques fixées dans les seules capitales, je ne connais pas d'ouvrage en langue française aussi bien documenté sur les tapas océaniens que celui-ci, réalisé à l'occasion de l'exposition homonyme organisée au musée de Cahors Henri-Martin en 2009. En le lisant, on n'ignore plus rien ni de la matière première, ni des techniques de fabrication, ni des fonctions assignées, ni des variantes culturelles, d'une extrémité à l'autre du domaine géographique qui lui est attaché. La clé du succès est probablement l'intelligence du commissaire de l'exposition, Laurent Guillaut, à avoir su recourir aux spécialistes du domaine exposé, quand bien même l'idée d'organiser une telle exposition dérivait d'une décision provinciale. Tout y est traité à la perfection, à la fois les textes et leurs illustrations, toutes en couleur, ce qui montre qu'en matière de reproductions artistiques, il est impossible d'échapper à cette exigence minimale. Mieux vaut ne rien éditer qu'éditer en noir et blanc un objet en couleur. 
Les spécialistes du domaine sont en l'occurrence Hélène Guiot et Fanny Wonu Veys, auxquelles ont été jointes les contributions tout aussi excellentes de Marie-Claire Bataille-Benguigui et Claude Stéfani. Hélène Guiot, en collaboration avec une association chargée de sa sauvegarde, réalise un inventaire de la plus importante collection de tapas polynésiens existant en Europe (plus de deux cents au musée océanien de La Neylière, près de Saint-Symphorien sur Coise). L'autre spécialiste, Wonu Veys, actuellement conservatrice des collections océaniennes au Museum voor Volkenkunde de Leiden aux Pays-Bas, a produit une remarquable thèse sur le tapa tongien et fidjien. On échappe du coup à l'écueil principal qui guette un tel type de production, à savoir une consommation esthétisante de l'œuvre privée de son contexte. Certes les critiques d'art peuvent offrir des clés de lecture intéressante de telles œuvres "d'art premier», mais des expert(e)s ayant séjourné durablement dans les îles productrices des œuvres ont forcément un point de vue mieux documenté que ceux qui n'y ont effectué que des séjours de courte durée, voire aucun séjour du tout.

Les chapitres de ce livre sont à la fois illustrés comme un catalogue, et documentés comme un véritable ouvrage d'analyse. On a donc en un seul ouvrage les avantages de deux genres habituellement distincts. L'effet est on ne peut plus bénéfique, joignant l'utile à l'agréable. L'agréable, c'est en l'occurrence la qualité des reproductions en couleur qui console le lecteur de n'avoir pu voir l'exposition et lui en restitue l'essentiel. L'utile ce sont les textes d'analyse qui accompagnent l'illustration, mais qui ne tombent pas dans le pédantisme, ou le discours abscons. Qu'on le veuille ou non, le meilleur commentaire possible sur des œuvres enracinées dans des terroirs qui continuent à les produire à l'heure actuelle, viendra toujours de gens ayant fréquenté les producteurs vivants de ces œuvres, et le commentaire sera encore meilleur si ces personnes sont des professionnelles du terrain et de la matière en débat.

Les six articles thématiques qui précèdent la présentation des objets du catalogue sont signés de MarieClaire Bataille-Benguigui, Hélène Guiot, Fanny Wonu Veys et Claude Stéfani. "Le «sens" des fibres en Océanie, écorce battue et autres végétaux» (pp. 7-16) de Marie-Claire Bataille-Benguigui est une entrée en matière à la fois dans la pratique et dans les représentations liées à la production du tapa en Océanie. Rappelant que le textile à base d'écorce battue est attesté dans toutes les zones tropicales du globe, l'auteur s'attache à démontrer sa spécificité océanienne. Elle commence par évoquer les mythes fondateurs associés à sa pratique, avant d'en recenser les usages les plus prestigieux.

La question de la matière première est aussi explicitée dans un bref article d'Hélène Guiot sur le cas concret de l'île Wallis ('Uvea). L'auteur nous détaille l'ensemble des matériaux utilisés dans la confection du tapa. Ceux-ci vont de l'écorce, plus exactement du liber qui en constitue la seconde couche, jusqu'à la colle, aux teintures et vernis employés, sans oublier les instruments mis à contribution dans cet art consommé de la transformation d'une ressource végétale.

Hélène Guiot revient dans un autre article consacré cette fois aux motifs wallisiens, et en particulier, à la forme « terre-mer » inaugurée au milieu de la décennie 1950 dans le contexte d'un développement de la production diligentée par les religieuses de la Congrégation des Sœurs Missionnaires de la Société de Marie. Elle nous fait découvrir les multiples aspects de ce décor du vingtième siècle qui n'a pas remplacé les autres formes de production, mais s'y est accolé. L'accumulation des formes semble en effet aller dans le sens de la «thésaurisation " justement relevée dans l'article de Marie-Claire Bataille-Benguigui.

La double contribution de Fanny Wonu Veys, qui a passé une année de recherche en résidance au musée du Quai Branly, reprend quelques-uns des thèmes développés dans sa remarquable thèse de doctorat. Dans la première "Le tapa : un emballage ordinaire sacré » (pp. 20-33), elle nous sensibilise au fait que la technique d'emballage se décline en de multiples fonctions, parfois antithétiques, qui vont de la protection à l'accueil du divin et de l'étranger, en passant par le voile et le dévoilement.

Dans la deuxième intitulée « Techniques de fabrication et de décoration » (pp. 34-41), elle prolonge sur le plan des pratiques ce que Hélène Guiot avait présenté sur le plan des matériaux. Elle nous fait saisir dans le détail les distinctions à établir entre les techniques d'immersion, d'enfumage, d'impression, de pochoir, de décoration à main levée, de matrices et de décor en filigrane. Son contexte de référence est emprunté aux Tonga et aux Fidji.

Le dernier article préparatoire au catalogue est de Claude Stéfani (pp. 50-54) qui s'intéresse à un tiputa, «manteau du roi de Bora-Bora » appartenant à la collection de l'ancienne École de médecine navale de Rochefort, pour en établir la véritable identification, et en affiner l'expertise à la lumière d'autres pièces provenant des îles de la Société.

Le catalogue proprement dit affiche 63 objets et il est signé de Laurent Guillaut, conservateur en chef du patrimoine au musée de Cahors Henri-Martin et commissaire de l'exposition, et de Fanny Wonu Veys qui a apporté son expertise à la documentation et à la présentation des objets exposés. Les objets sont bien entendu représentatifs de la diversité des lieux, des cultures, des matériaux et des décors produits. Ils proviennent notamment des collections des musées du Quai Branly de Paris et de La Neylière. Ils illustrent l'ensemble des archipels océaniens s'étalant d'ouest en est du Pacifique (le sens de circulation étant censé représenter le sens de la création historique et du flux des populations, échelle à la fois spatiale et temporelle) : Nouvelle-Guinée, Nouvelle-Bretagne, Santa Cruz, Vanuatu, Nouvelle-Calédonie, Fidji, Tonga, Samoa, Wallis, Futuna, Iles Marquises, Iles de la Société, Hawaii et Nouvelle-Zélande, bref un panorama on ne peut plus représentatif du domaine.

L'ensemble de l'ouvrage est servi par une iconographie impeccable, mais on retiendra autant la qualité des textes que celle des reproductions en couleur. Toutes ces raisons plaident au large recours à ce titre pour toutes les personnes désireuses d'en connaître sérieusement sur les tapas océaniens. S'il y avait une réserve à formuler, ce serait celle, somme toute 
mineure, du titre : « étoffes cosmiques » qui tendrait à donner un sens exagérément astronomique à cette production, alors qu'il suffit de les considérer comme des « biens de prestige » dont l'usage se manifeste au cours des moments les plus solennels de la vie sociale. Il ne nous viendrait pas à l'idée de traiter les tissus de la vie monarchique ou impériale française comme des « textiles cosmiques », quels que soient les éléments de décoration qui y apparaissent. L'emphase du titre mise à part, le livre doit désormais être classé dans la catégorie des ouvrages de référence sur la question du tapa en Océanie.

Raymond MAYER, Université Omar Bongo de Libreville et Université de Lyon 2

ZanetTe Didier, 2010. Le bestiaire mélanésien. 100 représentations, Nouméa, DZ Productions, «Et si nous parlions l'Océanien? », 160 p., bibliogr. indicative, une carte, table des 100 objets reproduits, lexique, nombreuses ill. couleur.

Sujet intéressant s'il en est mais mal illustré dans cet ouvrage où les photographies, ne sont pas toujours de qualité et montrent mal les objets, et dont le texte qui les accompagnent manquent de références à la bibliographie existante sur le sujet. La bibliographie, qui ne comporte que quinze références, est quasiment entièrement consacrée à " l'art » (huit des ouvrages mentionnés contiennent le mot art dans leur titre) ou aux collections muséales. Peu de références ethnographiques alors que le sujet ne peut être appréhendé à mon sens en faisant l'économie des apports de l'ethnologie, de l'ethnolinguistique et de l'importance des traditions orales en la matière. Puis, au fil de la lecture, on découvre en notes de bas de page quelques autres références qui seraient venues utilement compléter la bibliographie.

L'ouvrage est construit en sept chapitres faisant suite à l'introduction pp. 7-11): chapitre I «Aux débuts, les mythes... » (pp. 13-19); chapitre II « Dans l'océan » (pp. 21-45); chapitre III « Entre eaux saumâtres et terre » (pp. 47-65); chapitre IV « Sur terre, le roi cochon et les autres » (pp. 67-93); chapitre $\mathrm{V} « \mathrm{Au}$ pays des insectes» (pp. 95-101); chapitre VI «Le monde de la gent à plume » (pp. 103-131); chapitre VII «Et aujourd'hui?» (pp. 133-139), un index d'images (pp. 141-151) qui n'est en réalité qu'une table faite à l'aide de photos miniatures renvoyant à la page où l'objet est reproduit dans l'ouvrage et un glossaire (pp. 153-155). Pour finir, une page de références bibliographiques et un sommaire très peu indicatif qui est là en fait pour signaler les crédits photos, les artistes contemporains et les diverses mentions légales de l'ouvrage (un vrai fourre-tout).

Chaque chapitre commence par la reproduction d'une œuvre d'art contemporaine dont on trouve l'auteur dans le sommaire mais sans aucune précision ni de titre, ni de la nature de l'œuvre et encore moins sur l'artiste lui-même ! Dommage !

La page de préface pour introduire « l'importance de l'animal dans le bestiaire océanien » (p. 7) signée par Dominique Franchot (dont on ne nous dit pas qui il/elle est ${ }^{25}$ ) est bien limitée et nous place face à des lieux communs quand ce n'est pas des imprécisions pouvant conduire à de fausses interprétations, comme nous le verrons ci-dessous. L'introduction qui suit (pp. 7-11) ne nous satisfait guère plus. Ainsi, une telle phrase dans le premier paragraphe n'a guère de signification :

«En Nouvelle-Calédonie, on ne trouve plus, par exemple, qu'une espèce de mammifère la roussette et plus aucun reptile terrestre à l'exception toutefois du lézard. » (p. 7)

Ainsi, on ne sait pas à quelle période l'auteur fait référence avec ce « plus ». Si l'on se reporte au magnifique site internet endemia.nc, on trouve le serpent des Loyauté (Candoia bibroni) dit aussi boa du Pacifique et qui est un reptile terrestre (http://www.endemia. nc/faune/fiche1097.html - consulté le 3 avril 2010); côté lézard, leur variété endémique est immense. En $2003^{26}$, une recherche sur le site http://www.endemia. nc/ tel qu'il existait à cette date m'avait permis de recenser 67 espèces de lézards dont 59 endémiques, qui se répartissent en 42 espèces de Scincidae (dont 39 endémiques) et 25 espèces de Geckos (dont 20 endémiques appartenant à la famille Diplodactylidae qui comprend le genre Rhacodactylus cité par Leenhardt ; seules les cinq espèces de la famille Gekkonidae ne sont pas endémiques). Une telle phrase ne peut donc pas en rendre compte et laisser penser au lecteur non connaisseur que la faune calédonienne est d'une grande pauvreté, alors que quiconque s'intéresse à la Nouvelle-Calédonie en connaît la richesse endémique exceptionnelle.

En fait, chaque chapitre, après un paragraphe d'une banale généralité, ne fait que commenter les objets reproduits, sans forcément de liens entre les uns et les autres.

Beaucoup d'imprécisions, aucune information sur les objets collectés par l'auteur pour lesquels il aurait été utile de savoir où précisément il avait été trouvé, à quelle date et comment s'est faite l'acquisition et sur son usage particulier. Par exemple, l'objet 11, nommé «amulette» et dit «charme particulier» pour la pêche au dugong, nous est présenté sans autre précision sur les techniques de pêche de la région!

« Le lézard n'est pas toujours un ancêtre. Il est également l'objet de multiples légendes et de nombreux contes » (p. 65)

25. « Dominique Franchot était le directeur des Ressources humaines du Groupe ERAMET jusqu'en décembre 2009, c'est un passionné de voyages et d'art premier. Ses fonctions l'amenaient à venir régulièrement en Nouvelle-Calédonie pour la Société Le Nickel » (Isabelle Zanette, com. pers., 30/09/2010).

26. Voir la communication « Animaux, représentations totémiques et esprits des ancêtres dans quelques sociétés kanak de Nouvelle-Calédonie " que j'ai faite au colloque international le symbolisme des animaux - l'animal "clé de voûte " dans la tradition orale et les interactions homme-nature, organisé par le LACITO CNRS et l'IRD, à Villejuif (12-14/11/2003). 